CUBO A Mathematical Journal Vol.16, Noㅇ, (11-19). October 2014

\title{
A Note on Inextensible Flows of Curves on Oriented Surface
}

\author{
ONDER GOKMEN YILDIZ \\ Department of Mathematics, \\ Faculty of Arts and Sciences, \\ Bilecik Seyh Edebali \\ University, \\ Bilecik/TURKEY \\ ogokmen.yildiz@bilecik.edu.tr \\ SOLEY ERSOY \\ Department of Mathematics, \\ Faculty of Arts and Sciences, \\ Sakarya University, \\ Sakarya/TURKEY \\ sersoy@sakarya.edu.tr
}

\author{
Melek Masal \\ Department of Mathematics \\ Teaching, \\ Faculty of Education, \\ Sakarya University, \\ Sakarya/TURKEY \\ mmasal@sakarya.edu.tr
}

\begin{abstract}
In this paper, we investigate a general formulation for inextensible flows of curves on an oriented surface in $\mathbb{R}^{3}$. We obtain necessary and sufficient conditions as partial differential equations involving the geodesic curvature and the geodesic torsion for inextensible curve flow lying on an oriented surface. Moreover, some special cases of inextensible curves on oriented surface are given.
\end{abstract}

\section{RESUMEN}

En este artículo investigamos una formulación general para flujos inextensibles de curvas sobre una superficie orientable en $\mathbb{R}^{3}$. Obtenemos condiciones necesarias y suficientes para las ecuaciones diferenciales parciales que involucran la curva geodésica y la torsión geodésica para curvas inextensibles fluyendo sobre superficies orientadas. Más aún, se entregan algunos casos especiales de curvas inextensibles sobre superficies orientadas.

Keywords and Phrases: Curvature flows, inextensible, oriented surface.

2010 AMS Mathematics Subject Classification: 53C44, 53A04, 53A05. 


\section{Introduction}

The flow of the curve is said to be inextensible if its arclength preserved. Curve design using splines is one of the most fundamental topic in CAGD. Inextensible flows of the curves have beautiful shapes preserving connection to their control polygon. On the other hand, physically inextensible curve and surface flows give rise to motion which no strain energy is induced. For example, the swinging motion of a cord of fixed length can be described by inextensible curve and surface flows. Many authors have studied geometric flow problems and applications of inextensible curve flows, [1]-10]. An evolution equation for inelastic planar curves was derived by [9] and also, the general formulation of inextensible flows of curves and developable surfaces in $\mathbb{R}^{3}$ was exposed by [10].

In this paper, we derive a general formulation for inextensible flows of curves according to Darboux frame in $\mathbb{R}^{3}$. We give the necessary and sufficient conditions for an inextensible curve flow are expressed as a partial differential equations involving the geodesic curvature and geodesic torsion.

\section{Preliminaries}

Let $S$ be an oriented surface in three-dimensional Euclidean space $E^{3}$ and $\alpha(s)$ be a curve lying on the surface $S$. Suppose that the curve $\alpha(s)$ is spatial then there exists the Frenet frame $\{\vec{T}, \vec{N}, \vec{B}\}$ at each points of the curve where $\vec{T}$ is unit tangent vector, $\vec{N}$ is principal normal vector and $\vec{B}$ is binormal vector, respectively. The Frenet equation of the curve $\alpha(s)$ is given by

$$
\begin{aligned}
& \overrightarrow{\mathrm{T}^{\prime}}=\kappa \overrightarrow{\mathrm{N}} \\
& \overrightarrow{\mathrm{N}^{\prime}}=-\kappa \overrightarrow{\mathrm{T}}+\tau \overrightarrow{\mathrm{B}} \\
& \overrightarrow{\mathrm{B}^{\prime}}=-\tau \overrightarrow{\mathrm{N}}
\end{aligned}
$$

where $\mathrm{K}$ and $\tau$ are curvature and torsion of the curve $\alpha(\mathrm{s})$, respectively.

Since the curve $\alpha(\mathrm{s})$ lies on the surface $S$ there exists another frame of the curve $\alpha(\mathrm{s})$ which is called Darboux frame and denoted by $\{\vec{T}, \vec{g}, \vec{n}\}$. In this frame $\vec{T}$ is the unit tangent of the curve, $\vec{n}$ is the unit normal of the surface $S$ and $\vec{g}$ is a unit vector given by $\vec{g}=\vec{n} \times \vec{T}$. Since the unit tangent $\vec{T}$ is common element of both Frenet frame and Darboux frame, the vectors $\vec{N}, \vec{B}, \vec{g}$ and $\vec{n}$ lie on the same plane. So that the relations between these frames can be given as follows

$$
\left[\begin{array}{c}
\vec{T} \\
\vec{g} \\
\vec{n}
\end{array}\right]=\left[\begin{array}{ccc}
1 & 0 & 0 \\
0 & \cos \varphi & \sin \varphi \\
0 & -\sin \varphi & \cos \varphi
\end{array}\right]\left[\begin{array}{c}
\vec{T} \\
\vec{N} \\
\vec{B}
\end{array}\right]
$$

where $\varphi$ is the angle between the vectors $\vec{g}$ and $\vec{N}$. The derivative formulae of the Darboux frame 
is

$$
\left[\begin{array}{c}
\dot{\vec{T}} \\
\dot{\vec{g}} \\
\dot{\vec{n}}
\end{array}\right]=\left[\begin{array}{ccc}
0 & k_{g} & k_{n} \\
-k_{g} & 0 & \tau_{g} \\
-k_{n} & -\tau_{g} & 0
\end{array}\right]\left[\begin{array}{c}
\vec{T} \\
\vec{g} \\
\vec{n}
\end{array}\right]
$$

where $k_{g}, k_{n}$ and $\tau_{g}$ are called the geodesic curvature, the normal curvature and the geodesic torsions, respectively. Here and in the following, we use "dot" to denote the derivative with respect to the arc length parameter of a curve.

The relations between the geodesic curvature, normal curvature, geodesic torsion and $\kappa, \tau$ are given as follows, [1]

$$
k_{\mathrm{g}}=\kappa \cos \varphi, \quad \mathrm{k}_{\mathrm{n}}=-\mathrm{\kappa} \sin \varphi, \tau_{\mathrm{g}}=\tau+\frac{\mathrm{d} \varphi}{\mathrm{ds}} .
$$

Furthermore, the geodesic curvature $k_{g}$ and geodesic torsion $\tau_{g}$ of the curve $\alpha(s)$ can be calculated as follows, 11

$$
\begin{aligned}
& k_{g}=\left\langle\frac{d \vec{\alpha}}{d s}, \frac{d^{2} \vec{\alpha}}{d s^{2}} \times \vec{n}\right\rangle \\
& \tau_{g}=\left\langle\frac{d \vec{\alpha}}{d s}, \vec{n} \times \frac{d \vec{n}}{d s}\right\rangle .
\end{aligned}
$$

In the differential geometry of surfaces, for any curve $\alpha(s)$ lying on a surface $S$ the following relationships are well-known, [1]

i- $\alpha(s)$ is a geodesic curve if and only if $k_{g}=0$,

ii- $\alpha(s)$ is an asymptotic line if and only if $k_{n}=0$,

iii- $\alpha(\mathrm{s})$ is a principal line if and only if $\tau_{\mathrm{g}}=0$.

Through each point on a surface there passes, in general, a geodesic in every direction. A geodesic is uniquely determined by an initial point and tangent at that point. All straight lines on a surface are geodesics.

Along all curved geodesics the principal normal coincides with the surface normal. Along asymptotic lines osculating planes and tangent planes coincide, along geodesics they are normal. Through a point of a non-developable surface there are two asymptotic lines which can be real or imaginary.

\section{Inextensible Flows of Curve Lying on Oriented Surface}

Throughout this paper, we suppose that

$$
\alpha:[0, l] \times[0, w) \rightarrow M \subset E^{3}
$$

is a one parameter family of differentiable curves on orientable surface $M$ in $E^{3}$, where $l$ is the arclength of the initial curve. Let $\mathfrak{u}$ be the curve parameterization variable, $0 \leq \mathfrak{u} \leq \boldsymbol{l}$. If the speed of curve $\alpha$ is denoted by $v=\left\|\frac{\partial \vec{\alpha}}{\partial \mathfrak{u}}\right\|$ then the arclength of $\alpha$ is 


$$
S(u)=\int_{0}^{u}\left\|\frac{\partial \vec{\alpha}}{\partial u}\right\| d u=\int_{0}^{u} v d u .
$$

The operator $\frac{\partial}{\partial s}$ is given in terms of $u$ by

$$
\frac{\partial}{\partial s}=\frac{1}{v} \frac{\partial}{\partial u}
$$

Thus, the arclength is $\mathrm{d} s=v \mathrm{~d} u$.

Definition 3.1. Let $\mathrm{M}$ be an orientable surface and $\alpha$ be a differentiable curve on $\mathrm{M}$ in $\mathrm{E}^{3}$. Any flow of the curve $\alpha$ can be expressed with respect to Darboux frame $\{\overrightarrow{\mathrm{T}}, \overrightarrow{\mathrm{g}}, \overrightarrow{\mathrm{n}}\}$ in the following form:

$$
\frac{\partial \vec{\alpha}}{\partial t}=f_{1} \vec{T}+f_{2} \vec{g}+f_{3} \vec{n}
$$

Here, $f_{1}, f_{2}$ and $f_{3}$ are scalar speeds of the curve $\alpha$. Let the arclength variation be

$$
S(u, t)=\int_{0}^{u} v d u .
$$

In the Euclidean space the requirement that a curve not to be subject to any elongation or compression can be expressed by the condition

$$
\frac{\partial}{\partial t} S(u, t)=\int_{0}^{u} \frac{\partial v}{\partial t} d u=0, u \in[0,1]
$$

Definition 3.2. A curve evolution $\alpha(u, t)$ and its flow $\frac{\partial \vec{\alpha}}{\partial t}$ on the oriented surface $\mathrm{M}$ in $\mathrm{E}^{3}$ are said to be inextensible if

$$
\frac{\partial}{\partial t}\left\|\frac{\partial \vec{\alpha}}{\partial u}\right\|=0
$$

Now, we research the necessary and sufficient condition of a flow to be inextensible. For this reason, we need to the following Lemma.

Lemma 3.1. In $\mathrm{E}^{3}$, let $\mathrm{M}$ be an orientable surface and $\{\overrightarrow{\mathrm{T}}, \overrightarrow{\mathrm{g}}, \overrightarrow{\mathrm{n}}\}$ be a Darboux frame of $\alpha$ on $\mathrm{M}$. There exists following relation between the scalar speed functions $\mathrm{f}_{1}, \mathrm{f}_{2}, \mathrm{f}_{3}$ and the normal curvature $\mathrm{k}_{\mathrm{n}}$, geodesic curvature $\mathrm{k}_{\mathrm{g}}$ of $\alpha$ the curve

$$
\frac{\partial v}{\partial t}=\frac{\partial f_{1}}{\partial u}-f_{2} v k_{g}-f_{3} v k_{n}
$$


Proof. Since $\frac{\partial}{\partial u}$ and $\frac{\partial}{\partial t}$ commute and $v^{2}=\left\langle\frac{\partial \vec{\alpha}}{\partial u}, \frac{\partial \vec{\alpha}}{\partial u}\right\rangle$, we have

$$
\begin{aligned}
2 v \frac{\partial v}{\partial t} & =\frac{\partial}{\partial t}\left\langle\frac{\partial \vec{\alpha}}{\partial u}, \frac{\partial \vec{\alpha}}{\partial u}\right\rangle \\
& =2\left\langle\frac{\partial \vec{\alpha}}{\partial u}, \frac{\partial}{\partial u}\left(f_{1} \vec{T}+f_{2} \vec{g}+f_{3} \vec{n}\right)\right\rangle \\
& =2 v\left(\frac{\partial f_{1}}{\partial u}-f_{2} v k_{g}-f_{3} v k_{n}\right) .
\end{aligned}
$$

This completes the proof.

If we consider the conditions of being geodesic and asymptotic of a curve and Lemma 3.1, we can give the following corollary.

Corollary 3.1. If a curve is a geodesic curve or an asymptotic curve, then there are the following equations

$$
\frac{\partial v}{\partial t}=\frac{\partial f_{1}}{\partial u}-f_{3} v k_{n}
$$

or

$$
\frac{\partial v}{\partial t}=\frac{\partial f_{1}}{\partial u}-f_{2} v k_{g}
$$

respectively.

Theorem 3.1. Let $\{\vec{T}, \vec{g}, \vec{n}\}$ be the Darboux frame of a curve $\alpha$ on $M$ and $\frac{\partial \vec{\alpha}}{\partial t}=f_{1} \vec{T}+f_{2} \vec{g}+$ $\mathrm{f}_{3} \overrightarrow{\mathrm{n}}$ be a differentiable flow of $\alpha$ in $\mathbb{R}^{3}$. Then the flow is inextensible if and only if

$$
\frac{\partial f_{1}}{\partial s}=f_{2} k_{g}+f_{3} k_{n}
$$

Proof. Suppose that the curve flow is inextensible. From the equations (3.4) and (3.6) for $u \in[0, l]$ we see that

$$
\frac{\partial}{\partial t} S(u, t)=\int_{0}^{u} \frac{\partial v}{\partial t} d u=\int_{0}^{u}\left(\frac{\partial f_{1}}{\partial u}-f_{2} v k_{g}-f_{3} v k_{n}\right) d u=0 .
$$

Thus, it can be seen that

$$
\frac{\partial f_{1}}{\partial u}=f_{2} v k_{g}+f_{3} v k_{n} .
$$

Considering the last equation and (3.2), we reach

$$
\frac{\partial f_{1}}{\partial s}=f_{2} k_{g}+f_{3} k_{n} .
$$

Conversely, by following a similar way as above, the proof is completed.

From Theorem 3.1, we have following corollary. 
Corollary 3.2. $i$ - Let the curve $\alpha$ is a geodesic curve on $M$. Then the curve flow is inextensible if and only if $\frac{\partial \mathrm{f}_{1}}{\partial \mathrm{s}}=\mathrm{f}_{3} \mathrm{k}_{\mathrm{n}}$.

ii- Let the curve $\alpha$ is an asymptotic line on $\mathrm{M}$. Then the curve flow is inextensible if and only if $\frac{\partial \mathrm{f}_{1}}{\partial \mathrm{s}}=\mathrm{f}_{2} \mathrm{k}_{\mathrm{g}}$.

Now, we restrict ourselves to the arclength parameterized curves. That is, $v=1$ and the local coordinate $u$ corresponds to the curve arclength $s$. We require the following Lemma.

Lemma 3.2. Let $M$ be an orientable surface in $E^{3}$ and $\{\vec{T}, \vec{g}, \vec{n}\}$ be a Darboux frame of the curve $\alpha$ on $M$. Then, the differentiations of $\{\vec{T}, \vec{g}, \vec{n}\}$ with respect to $t$ is

$$
\begin{aligned}
& \frac{\partial \vec{T}}{\partial t}=\left(f_{1} k_{g}+\frac{\partial f_{2}}{\partial s}-f_{3} \tau_{g}\right) \vec{g}+\left(f_{1} k_{n}+\frac{\partial f_{3}}{\partial s}+f_{2} \tau_{g}\right) \vec{n} \\
& \frac{\partial \vec{g}}{\partial t}=-\left(f_{1} k_{g}+\frac{\partial f_{2}}{\partial s}-f_{3} \tau_{g}\right) \vec{T}+\psi \vec{n} \\
& \frac{\partial \vec{n}}{\partial t}=-\left(f_{1} k_{n}+\frac{\partial f_{3}}{\partial s}+f_{2} \tau_{g}\right) \vec{T}-\psi \vec{g}
\end{aligned}
$$

where $\psi=\left\langle\frac{\partial \vec{g}}{\partial t}, \vec{n}\right\rangle$.

Proof. Since $\frac{\partial}{\partial t}$ and $\frac{\partial}{\partial s}$ are commutative, it seen that

$$
\begin{aligned}
\frac{\partial \vec{T}}{\partial t} & =\frac{\partial}{\partial t}\left(\frac{\partial \vec{\alpha}}{\partial s}\right)=\frac{\partial}{\partial s}\left(\frac{\partial \vec{\alpha}}{\partial t}\right)=\frac{\partial}{\partial s}\left(f_{1} \vec{T}+f_{2} \vec{g}+f_{3} \vec{n}\right) \\
& =\frac{\partial f_{1}}{\partial s} \vec{T}+f_{1} \frac{\partial \vec{T}}{\partial s}+\frac{\partial f_{2}}{\partial s} \vec{g}+f_{2} \frac{\partial \vec{g}}{\partial s}+\frac{\partial f_{3}}{\partial s} \vec{n}+f_{3} \frac{\partial \vec{n}}{\partial s} .
\end{aligned}
$$

Substituting the equation (3.7) into the last equation and using Theorem 3.1, we have

$$
\frac{\partial \vec{T}}{\partial t}=\left(f_{1} k_{g}+\frac{\partial f_{2}}{\partial s}-f_{3} \tau_{g}\right) \vec{g}+\left(f_{1} k_{n}+\frac{\partial f_{3}}{\partial s}+f_{2} \tau_{g}\right) \vec{n}
$$

Now, let us differentiate the Darboux frame with respect to $t$ as follows;

$$
\begin{aligned}
0 & =\frac{\partial}{\partial t}\langle\vec{T}, \vec{g}\rangle=\left\langle\frac{\partial \vec{T}}{\partial t}, \vec{g}\right\rangle+\left\langle\vec{T}, \frac{\partial \vec{g}}{\partial t}\right\rangle \\
& =\left(f_{1} k_{g}+\frac{\partial f_{2}}{\partial s}-f_{3} \tau_{g}\right)+\left\langle\vec{T}, \frac{\partial \vec{g}}{\partial t}\right\rangle \\
0 & =\frac{\partial}{\partial t}\langle\vec{T}, \vec{n}\rangle=\left\langle\frac{\partial \vec{T}}{\partial t}, \vec{n}\right\rangle+\left\langle\vec{T}, \frac{\partial \vec{n}}{\partial t}\right\rangle \\
& =\left(f_{1} k_{n}+\frac{\partial f_{3}}{\partial s}+f_{2} \tau_{g}\right)+\left\langle\vec{T}, \frac{\partial \vec{n}}{\partial t}\right\rangle
\end{aligned}
$$

From (3.10) and (3.11), we have obtain

$$
\frac{\partial \vec{g}}{\partial t}=-\left(f_{1} k_{g}+\frac{\partial f_{2}}{\partial s}-f_{3} \tau_{g}\right) \vec{T}+\psi \vec{n}
$$


and

$$
\frac{\partial \vec{n}}{\partial t}=-\left(f_{1} k_{n}+\frac{\partial f_{3}}{\partial s}+f_{2} \tau_{g}\right) \vec{T}-\psi \vec{g}
$$

respectively, where $\psi=\left\langle\frac{\partial \vec{g}}{\partial t}, \vec{n}\right\rangle$.

If we take into consideration last Lemma, we have following corollary.

Corollary 3.3. Let $\mathrm{M}$ be an orientable surface in $\mathrm{E}^{3}$.

$i$ - If the curve $\alpha$ is a geodesic curve, then

$$
\begin{aligned}
& \frac{\partial \vec{T}}{\partial t}=\left(\frac{\partial f_{2}}{\partial s}-f_{3} \tau_{g}\right) \vec{g}+\left(f_{1} k_{n}+\frac{\partial f_{3}}{\partial s}+f_{2} \tau_{g}\right) \vec{n}, \\
& \frac{\partial \vec{g}}{\partial t}=-\left(\frac{\partial f_{2}}{\partial s}-f_{3} \tau_{g}\right) \vec{T}+\psi \vec{n}, \\
& \frac{\partial \vec{n}}{\partial t}=-\left(f_{1} k_{n}+\frac{\partial f_{3}}{\partial s}+f_{2} \tau_{g}\right) \vec{T}-\psi \vec{g},
\end{aligned}
$$

where $\psi=\left\langle\frac{\partial \vec{g}}{\partial t}, \vec{n}\right\rangle$.

ii- If the curve $\alpha$ is an asymptotic line, then

$$
\begin{aligned}
& \frac{\partial \vec{T}}{\partial t}=\left(f_{1} k_{g}+\frac{\partial f_{2}}{\partial s}-f_{3} \tau_{g}\right) \vec{g}+\left(\frac{\partial f_{3}}{\partial s}+f_{2} \tau_{g}\right) \vec{n}, \\
& \frac{\partial \vec{g}}{\partial t}=-\left(f_{1} k_{g}+\frac{\partial f_{2}}{\partial s}-f_{3} \tau_{g}\right) \vec{T}+\psi \vec{n}, \\
& \frac{\partial \vec{n}}{\partial t}=-\left(\frac{\partial f_{3}}{\partial s}+f_{2} \tau_{g}\right) \vec{T}-\psi \vec{g},
\end{aligned}
$$

where $\psi=\left\langle\frac{\partial \vec{g}}{\partial t}, \vec{n}\right\rangle$.

iii- If the curve is a curvature line, then

$$
\begin{aligned}
& \frac{\partial \vec{T}}{\partial t}=\left(f_{1} k_{g}+\frac{\partial f_{2}}{\partial s}\right) \vec{g}+\left(f_{1} k_{n}+\frac{\partial f_{3}}{\partial s}\right) \vec{n}, \\
& \frac{\partial \vec{g}}{\partial t}=-\left(f_{1} k_{g}+\frac{\partial f_{2}}{\partial s}\right) \vec{T}+\psi \vec{n}, \\
& \frac{\partial \vec{n}}{\partial t}=-\left(f_{1} k_{n}+\frac{\partial f_{3}}{\partial s}\right) \vec{T}-\psi \vec{g},
\end{aligned}
$$

where $\psi=\left\langle\frac{\partial \vec{g}}{\partial t}, \vec{n}\right\rangle$.

Theorem 3.2. Suppose that the curve flow $\frac{\partial \vec{\alpha}}{\partial t}=f_{1} \vec{T}+f_{2} \vec{g}+f_{3} \vec{n}$ is inextensible on the orientable surface on $\mathrm{M}$. In this case, the following partial differential equations are held:

$$
\begin{aligned}
& \frac{\partial k_{g}}{\partial t}=f_{2} k_{g}^{2}+f_{3} k_{g} k_{n}+f_{1} \frac{\partial k_{g}}{\partial s}+\frac{\partial^{2} f_{2}}{\partial s^{2}}-2 \frac{\partial f_{3}}{\partial s} \tau_{g}-f_{3} \frac{\partial \tau_{g}}{\partial s}-f_{1} k_{n} \tau_{g}-f_{2} \tau_{g}^{2}+\psi k_{n}, \\
& \frac{\partial k_{n}}{\partial t}=f_{2} k_{g} k_{n}+f_{3} k_{n}^{2}+f_{1} \frac{\partial k_{n}}{\partial s}+\frac{\partial^{2} f_{3}}{\partial s^{2}}+2 \frac{\partial f_{2}}{\partial s} \tau_{g}+f_{2} \frac{\partial \tau_{g}}{\partial s}+f_{1} k_{g} \tau_{g}-f_{3} \tau_{g}^{2}-\psi k_{g}, \\
& \frac{\partial \tau_{g}}{\partial t}=f_{2} k_{g} \tau_{g}-\frac{\partial f_{2}}{\partial s} k_{n}+\frac{\partial f_{3}}{\partial s} k_{g}+f_{3} k_{n} \tau_{g}+\frac{\partial \psi}{\partial s} .
\end{aligned}
$$

Proof. Since $\frac{\partial}{\partial s} \frac{\partial \vec{T}}{\partial t}=\frac{\partial}{\partial t} \frac{\partial \vec{T}}{\partial s}$ we get

$$
\begin{aligned}
\frac{\partial}{\partial s} \frac{\partial \vec{T}}{\partial t}= & \frac{\partial}{\partial s}\left[\left(f_{1} k_{g}+\frac{\partial f_{2}}{\partial s}-f_{3} \tau_{g}\right) \vec{g}+\left(f_{1} k_{n}+\frac{\partial f_{3}}{\partial s}+f_{2} \tau_{g}\right) \vec{n}\right] \\
= & \left(\frac{\partial f_{1}}{\partial s} k_{g}+f_{1} \frac{\partial k_{g}}{\partial s}+\frac{\partial^{2} f_{2}}{\partial s^{2}}-\frac{\partial f_{3}}{\partial s} \tau_{g}-f_{3} \frac{\partial \tau_{g}}{\partial s}\right) \vec{g}+\left(f_{1} k_{g}+\frac{\partial f_{2}}{\partial s}-f_{3} \tau_{g}\right) \frac{\partial \vec{g}}{\partial s} \\
& +\left(\frac{\partial f_{1}}{\partial s} k_{n}+f_{1} \frac{\partial k_{n}}{\partial s}+\frac{\partial^{2} f_{3}}{\partial s^{2}}+\frac{\partial f_{2}}{\partial s} \tau_{g}+f_{2} \frac{\partial \tau_{g}}{\partial s}\right) \vec{n}+\left(f_{1} k_{n}+\frac{\partial f_{3}}{\partial s}+f_{2} \tau_{g}\right) \frac{\partial \vec{n}}{\partial s}
\end{aligned}
$$


i.e.,

$$
\begin{aligned}
\frac{\partial}{\partial s} \frac{\partial \vec{T}}{\partial t}= & \left(\frac{\partial f_{1}}{\partial s} k_{g}+f_{1} \frac{\partial k_{g}}{\partial s}+\frac{\partial^{2} f_{2}}{\partial s^{2}}-\frac{\partial f_{3}}{\partial s} \tau_{g}-f_{3} \frac{\partial \tau_{g}}{\partial s}\right) \vec{g}+\left(f_{1} k_{g}+\frac{\partial f_{2}}{\partial s}-f_{3} \tau_{g}\right)\left(-k_{g} \vec{T}+\tau_{g} \vec{n}\right) \\
& +\left(\frac{\partial f_{1}}{\partial s} k_{n}+f_{1} \frac{\partial k_{n}}{\partial s}+\frac{\partial^{2} f_{3}}{\partial s^{2}}+\frac{\partial f_{2}}{\partial s} \tau_{g}+f_{2} \frac{\partial \tau_{g}}{\partial s}\right) \vec{n}+\left(f_{1} k_{n}+\frac{\partial f_{3}}{\partial s}+f_{2} \tau_{g}\right)\left(-k_{g} \vec{T}-\tau_{g} \vec{g}\right)
\end{aligned}
$$

while

$$
\frac{\partial}{\partial t} \frac{\partial \vec{T}}{\partial s}=\frac{\partial}{\partial t}\left(k_{g} \vec{g}+k_{n} \vec{n}\right)=\frac{\partial k_{g}}{\partial t} \vec{g}+k_{g} \frac{\partial \vec{g}}{\partial t}+\frac{\partial k_{n}}{\partial t} \vec{n}+k_{n} \frac{\partial \vec{n}}{\partial t} .
$$

Thus, from the both of above two equations, we reach

$$
\frac{\partial k_{g}}{\partial t}=f_{2} k_{g}^{2}+f_{3} k_{g} k_{n}+f_{1} \frac{\partial k_{g}}{\partial s}+\frac{\partial^{2} f_{2}}{\partial s^{2}}-2 \frac{\partial f_{3}}{\partial s} \tau_{g}-f_{3} \frac{\partial \tau_{g}}{\partial s}-f_{1} k_{n} \tau_{g}-f_{2} \tau_{g}^{2}+\psi k_{n}
$$

and

$$
\frac{\partial k_{n}}{\partial t}=f_{2} k_{g} k_{n}+f_{3} k_{n}^{2}+f_{1} \frac{\partial k_{n}}{\partial s}+\frac{\partial^{2} f_{3}}{\partial s^{2}}+2 \frac{\partial f_{2}}{\partial s} \tau_{g}+f_{2} \frac{\partial \tau_{g}}{\partial s}+f_{1} k_{g} \tau_{g}-f_{3} \tau_{g}^{2}-\psi k_{g} .
$$

Noting that $\frac{\partial}{\partial s} \frac{\partial \vec{g}}{\partial t}=\frac{\partial}{\partial t} \frac{\partial \vec{g}}{\partial s}$, it is seen that

$$
\begin{aligned}
\frac{\partial}{\partial s} \frac{\partial \vec{g}}{\partial t}= & \frac{\partial}{\partial s}\left[-\left(f_{1} k_{g}+\frac{\partial f_{2}}{\partial s}-f_{3} \tau_{g}\right) \vec{T}+\psi \vec{n}\right] \\
= & -\left(\frac{\partial f_{1}}{\partial s} k_{g}+f_{1} \frac{\partial k_{g}}{\partial s}+\frac{\partial^{2} f_{2}}{\partial s^{2}}-\frac{\partial f_{3}}{\partial s} \tau_{g}-f_{3} \frac{\partial \tau_{g}}{\partial s}\right) \vec{T} \quad \text { while } \\
& -\left(f_{1} k_{g}+\frac{\partial f_{2}}{\partial s}-f_{3} \tau_{g}\right)\left(k_{g} \vec{g}+k_{n} \vec{n}\right) \\
& +\frac{\partial \psi}{\partial s} n+\psi\left(-k_{n} \vec{T}-\tau_{g} \vec{g}\right) \\
& \frac{\partial}{\partial t} \frac{\partial \vec{g}}{\partial s}=\frac{\partial}{\partial t}\left(-k_{g} \vec{T}+\tau_{g} \vec{n}\right)=-\frac{\partial k_{g}}{\partial t} \vec{T}-k_{g} \frac{\partial \vec{T}}{\partial t}+\frac{\partial \tau_{g}}{\partial t} \vec{n}+\tau_{g} \frac{\partial \vec{n}}{\partial t} .
\end{aligned}
$$

Thus, we obtain

$$
\frac{\partial \tau_{g}}{\partial t}=f_{2} k_{g} \tau_{g}-\frac{\partial f_{2}}{\partial s} k_{n}+\frac{\partial f_{3}}{\partial s} k_{g}+f_{3} k_{n} \tau_{g}+\frac{\partial \psi}{\partial s} .
$$

No other new formulas are obtained from the relation $\frac{\partial}{\partial s} \frac{\partial \vec{n}}{\partial t}=\frac{\partial}{\partial t} \frac{\partial \vec{n}}{\partial s}$.

Thus, we give the following corollary from last theorem.

Corollary 3.4. Let $\mathrm{M}$ be an orientable surface in $\mathrm{E}^{3}$.

$i$-If the curve $\alpha$ is a geodesic curve on $\mathrm{M}$, then we have

$$
\frac{\partial k_{n}}{\partial t}=f_{3} k_{n}^{2}+f_{1} \frac{\partial k_{n}}{\partial s}+\frac{\partial^{2} f_{3}}{\partial s^{2}}+2 \frac{\partial f_{2}}{\partial s} \tau_{g}+f_{2} \frac{\partial \tau_{g}}{\partial s}-f_{3} \tau_{g}^{2}
$$

and

$$
\frac{\partial \tau_{g}}{\partial t}=-\frac{\partial f_{2}}{\partial s} k_{n}+f_{3} k_{n} \tau_{g}+\frac{\partial \psi}{\partial s} .
$$

ii- If the curve $\alpha$ is an asymptotic line, we have

$$
\frac{\partial k_{g}}{\partial t}=f_{2} k_{g}^{2}+f_{1} \frac{\partial k_{g}}{\partial s}+\frac{\partial^{2} f_{2}}{\partial s^{2}}-2 \frac{\partial f_{3}}{\partial s} \tau_{g}-f_{3} \frac{\partial \tau_{g}}{\partial s}-f_{2} \tau_{g}^{2}
$$


and

$$
\frac{\partial \tau_{g}}{\partial t}=f_{2} k_{g} \tau_{g}+\frac{\partial f_{3}}{\partial s} k_{g}+\frac{\partial \psi}{\partial s} .
$$

iii- If the curve $\alpha$ is a curvature line, then we have

$$
\begin{gathered}
\frac{\partial k_{g}}{\partial t}=f_{2} k_{g}^{2}+f_{3} k_{g} k_{n}+f_{1} \frac{\partial k_{g}}{\partial s}+\frac{\partial^{2} f_{2}}{\partial s^{2}}+\psi k_{n} \\
\frac{\partial k_{n}}{\partial t}=f_{2} k_{g} k_{n}+f_{3} k_{n}^{2}+f_{1} \frac{\partial k_{n}}{\partial s}+\frac{\partial^{2} f_{3}}{\partial s^{2}}-\psi k_{g} . \\
\text { Received: September 2013. Accepted: May } 2014 .
\end{gathered}
$$

\section{References}

[1] G. Chirikjian, J. Burdick, A modal approach to hyper-redundant manipulator kinematics, IEEE Trans. Robot. Autom. 10 (1994), 343-354.

[2] H. Mochiyama, E. Shimemura, H. Kobayashi, Shape control of manipulators with hyper degrees of freedom, Int. J. Robot.Res., 18 (1999), 584-600.

[3] M. Kass, A. Witkin, D. Terzopoulos, Snakes: active contour models, in: Proc. 1st Int. Conference on Computer Vision (1987), 259-268.

[4] H.Q. Lu, J.S. Todhunter, T.W. Sze, Congruence conditions for nonplanar developable surfaces and their application to surface recognition, CVGIP, Image Underst. 56 (1993), 265-285.

[5] M. Desbrun, M.-P. Cani-Gascuel, Active implicit surface for animation, in: Proc. Graphics Interface-Canadian Inf. Process. Soc. (1998), 143-150.

[6] D.J. Unger, Developable surfaces in elastoplastic fracture mechanics, Int. J. Fract. 50 (1991), 33-38.

[7] M. Gage, R.S. Hamilton, The heat equation shrinking convex plane curves, J. Differential Geom. 23 (1986), 69-96.

[8] M. Grayson, The heat equation shrinks embedded plane curves to round points, J. Differential Geom. 26(1987), 285-314.

[9] D.Y. Kwon, F.C. Park, Evolution of inelastic plane curves, Appl. Math. Lett., 12 (1999), 115-119.

[10] D.Y. Kwon, F.C. Park, D.P. Chi, Inextensible flows of curves and developable surfaces, Appl. Math. Lett., 18 (2005), 1156-1162.

[11] B. O’Neill, Elementary Differential Geometry, Academic Press Inc. New York, 1966. 